\title{
The Evolution of Ellipticals, Spirals and Irregulars: Overcoming Selection Bias
}

\author{
Simon Driver \\ University of St Andrews, St Andrews, KY16 9DW, SCOTLAND
}

\begin{abstract}
The Hubble Deep Fields represent our best opportunity for probing galaxy evolution over a substantive look-back time. However as with any dataset the HDFs are prone to selection biases. These biases are extremely severe beyond $z \sim 1.25$ such that a meaningful interpretation of generic galaxy evolution is not possible. We can however extract well defined volume-limited samples at $z<1$. The data are entirely consistent with passive/null-evolution for ellipticals, spirals and irregulars however this concluion is tempered by small number statistics. Alas stringent constraints on galaxy evolution await an order of magnitude increase in the number of HDFs.
\end{abstract}

\section{Introduction}

The two Hubble Deep Fields (HDF) provide our deepest optical insight into the near and far universe. Each contains approximately 750 galaxies for which magnitudes, colours, photometric redshifts and morphologies have been determined [1]. The data have been used by many groups to construct a picture of the assembly and evolution of galaxies. However every dataset, the HDFs included, suffer from selection biases at some level. Here I hope to briefly highlight the severity of these selection biases and to provide some suggestions as to how to test for, and monitor, these biases. In particular I advocate the use of the Bivariate Brightness Distribution (BBD) as a means of probing galaxy evolution while also tracking the biases which plague our ability to decipher deep data. Finally I put forward my own cautious conclusions as to the evolution of ellipticals, spirals and irregulars since $\mathrm{z}=1$.

\section{Selection Bias}

Galaxies exist over a vast dynamic range in both luminosity and in surface brightness. Any dataset has an apparent magnitude limit, a surface brightness detection limit and size limits which together create a selection window [2]. We view of the galaxy population through this selection window Different surveys with differing limitations will sample different regions of the galaxian parameter-space. Unless these biases are monitored discrepancies will manifest between surveys as are seen for example in measures of the local luminosity function [3]. These concerns become more paramount when 
one compares local and deep data, as such biases will cause discrepancies that may erroneously be interpreted as evolution. Additionally as these selection biases are all distance dependent the selection window varies such that comparisons within a single dataset are also susceptible. The most important of these biases is Malmquist Bias - i.e., as we go fainter we select against firstly, low luminosity systems, then normal systems and eventually giant systems. An analogous bias, Disney Bias [4], works in a similar way but in terms of a galaxy's absolute surface brightness such that lower surface brightness systems are preferentially lost first, etc. Neglecting either Malmquist or Disney Bias leads to misleading results and as both biases are ever present it is vital that we, as a community, demonstrate the robustness of our data to these biases prior to interpretation. Fig. 1 shows two visualisations of the HDF data highlighting the severity of both the Malmquist bias (left) and the Disney bias (right). The dashed lines shows the location of the canonical non-evolving zero-redshift $L_{*}$ point.
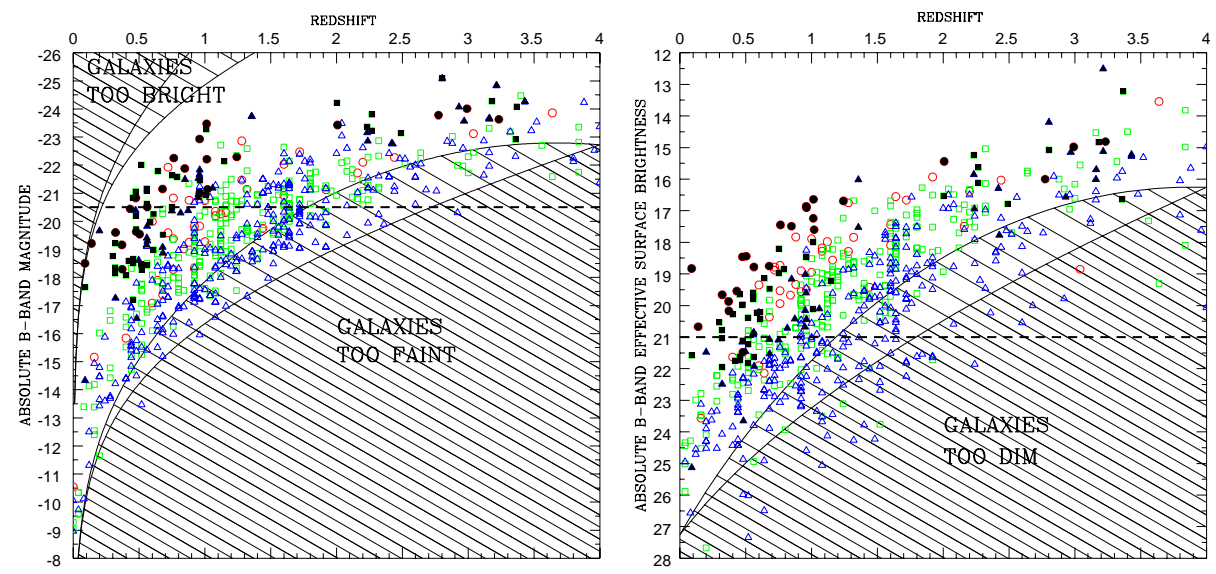

Fig. 1. These two plots ( $M \mathrm{v} z$, left) and ( $\mu \mathrm{v} z$, right) highlight the severe effect of Malmquist- and Disney- Bias which impinge define the selection window through which we view the galaxy population at any redshift. The two selection limits define the uncertainty due to the range of possible K-corrections. Solid points are spectrscopic redshifts, circles are ellipticals, squares spirals and triangles irregulars

The conclusion we must draw from Fig. 11 is that the HDF suffers from severe bias beyond $\mathrm{z}=1$ (for the spectroscopic sample:solid points) and beyond $\mathrm{z}=1.25$ (for the photometric sample). Any statistical measurement of luminosity functions, star-formation history, evolution etc, will therefore be incomplete and increasingly misleading beyond $\mathrm{z}=1.25$. So, does the mean 
star-formation of the Universe really peak at $\mathrm{z}=1.5$ and then turn down ? Fig. 1 convinces me that we simply don't know until we look into the "unobserved" regions. Looking at Fig. 1 it is also easy to see why one might be drawn to the conclusion that galaxies were both more luminous and of higher surface brightness in the past. Again an alternative and more informed conclusion is that this interpretation may be purely due to the shifting selection window - we can not say.

The conclusion is that the Hubble Deep Fields lack the breadth in luminosity and surface brightness beyond $\mathrm{z}=1.25$ to place meaningful constraints on galaxy evolution.

\section{The Bivariate Brightness Distribution}

Having concluded that a statistical investigation of galaxy evolution from the HDFs is currently impossible beyond $\mathrm{z}=1.25$ this confines us to investigation galaxy evolution at $z<1.25$. This is not to say that selection biases are not present below $z<1.25$ but that they have not yet impinged upon the canonical $\mathrm{z}=0 L_{*}$ point. Why is the $L_{*}$ point so critical ? mainly because it is these galaxies which dominate the luminosity and mass density of the local universe [5]. To see where and whether they have to evolved we have to be able to "observe" any positive-, zero- and negative- evolution about this point.

Additional selection lines also exist which cannot be shown on Fig. 政 These are the size constraints due to the pixel size (limiting the highest measurable surface brightness) and the field-of-view size (limiting the lowest surface brightness object detectable for any absolute magnitude at some redshift). To show these additional selection lines we must simultaneously look at the $M-\mu$ plane (our window into the galaxy population). This plane is known as the Bivariate Brightness Distribution and has up until no only be derived for the Virgo cluster [6] - nevertheless it is a powerful tool.

An example BBD, taken from [2], is shown as Fig. 2. This shows the HDF window into the galaxy population at $\mathrm{z}=0.3-0.5$. The absolute magnitude, surface brightness and size selection lines are all shown and the shaded regions indicate areas where the HDF is unable to detect galaxies. The selection lines are all redshift dependent and control our insight into the galaxy population. The selection lines are fairly straight-forward to estimate and are laid out in detail in [2]. Note that those galaxies which appear in the excluded region must have erroneous photometric redshifts as their detection is impossible. As an aside the data show a strong luminosity-surface brightness relation and a dearth of luminous low surface brightness galaxies [2].

Using a bivariate brightness distribution we can extract volumelimited samples for which the selection biases are well defined. 


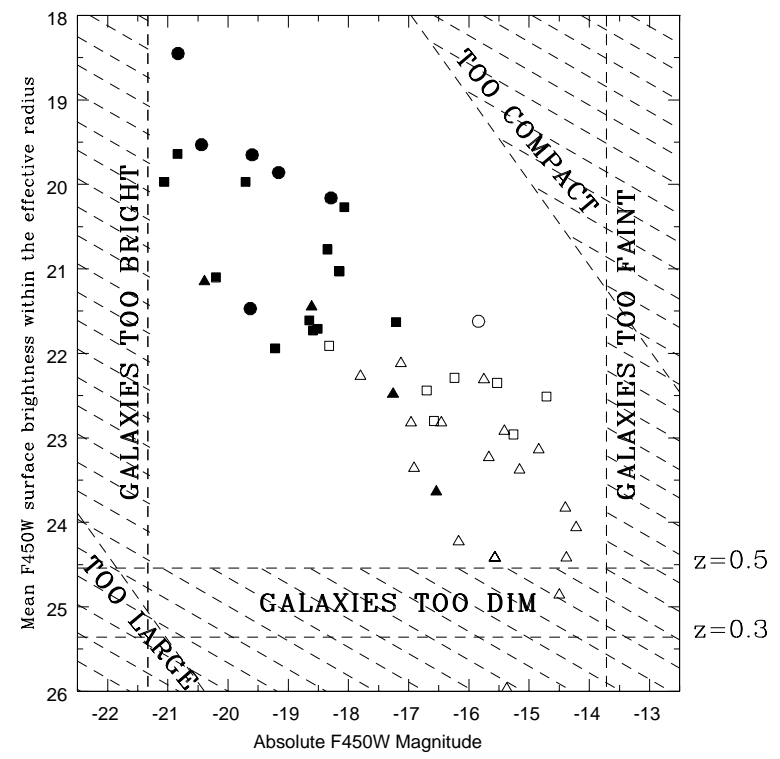

Fig. 2. The bivariate brightness distribution for a volume limited $(0.3 ; \mathrm{z} ; 0.5)$ sample showing the various selection lines at work.

\section{The Evolution of Ellipticals, Spirals and Irregulars}

Having advocated a methodology to extract well controlled samples below $z=1.25$, we are now in a good position to investigate galaxy evolution since $z=1$. Fig. 3 shows a sequence of BBDs for all the HDF ellipticals, spirals and irregulars from $\mathrm{z}=0$ to $\mathrm{z}=1$ for $B<26.5$. The striped areas show the prohibited regions and galaxies that lie within these regions most likely have erroneous photometric redshifts. The density of galaxies which lie in these excluded regions is of some concern and highlights the desperate need to spectroscopically confirm late-types in particular.

Finally then what does Fig. 3 tell us about the evolution of the three generic galaxy types. The dashed line guides the eye and are fitted to the $\mathrm{z}=0.3-0.6$ bin for each type. Two conclusions strike home: (1) the HDF contain insufficient data for a detailed statistical analysis particularly when one folds in the clustering uncertainty, and (2), in all cases the 0.3-0.6 fit matches the 0.8-1.0 fit.

In closing it appears that the current outlook is somewhat depressing, the HDF is insufficiently deep to probe the evolution of the generic population beyond $z \sim 1.25$ and the volume insufficient to probe evolution below $z \sim$ 1.25. We desperately need an order of magnitude more HDFs. 

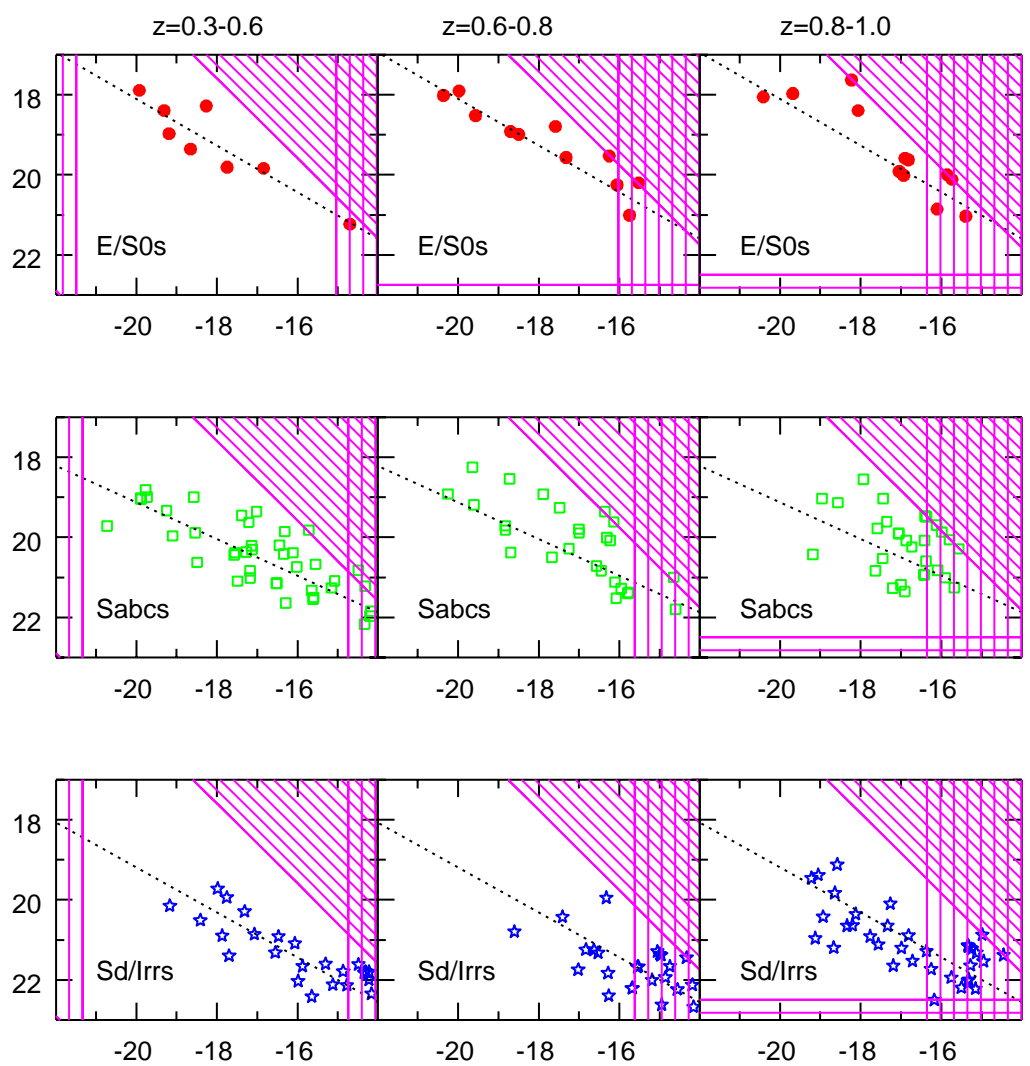

Fig. 3. The BBD plots for ellipticals (top), spirals (middle) and irregulars (bottom) for redshift intervals of 0.3-0.6 (left), 0.6-0.8 (centre), 0.8-1.0 (right)

For the moment the Occam's razor solution must surely prevail: within the limited statistics available the HDF is consitent with null/passive evolution since $z=1$, beyond this even the HDF data are dominated by selection effects.

\section{References}

1. Driver, S.P., et al., (1998) ApJL, 496, L93

2. Driver, S.P., (2000) ApJL, 526, L69

3. Cross, N.J., Driver, S.P., (2001) ApJL, submitted

4. Disney, M.J., (1976) Nature, 263, 573

5. Cross, N.J., (2000) MNRAS, in press

6. Ferguson, H., Binggeli, B., (1994) A\&ARv, 6, 67 